\title{
The Microbiologically Influenced Corrosion Behavior of C-Mn Ship Structural Steel with Different Manufacturing Processes
}

\author{
Hwan Gyo JUNG, Jang Yong YOO and Jong Soo WOO \\ POSCO, Technical Research Laboratories, Pohang, Korea. \\ (Received on November 29, 2002; accepted in final form on April 14, 2003)
}

\begin{abstract}
The MIC (Microbiologically Influenced Corrosion) of lower deck steel plates in double hull VLCC (Very Large Crude Oil Carrier) cargo tanks has been focused because of severe localized corrosion. Recently, ship companies have reported that TMCP (Thermo-mechanical Control Process) steel plates have been showed more severe localized corrosion than conventional rolled steels. In order to elucidate the MIC resistance of TMCP steels by comparison with conventional rolled steels and normalized steels in environments of double hull VLCC cargo oil tanks, various measurements and corrosion tests were performed such as measurements of polarization curves, immersion tests in bacteria culture medium.

All results revealed that three types of steels have almost the same corrosion resistance in bacteria culture medium. Three kinds of steel exhibit almost the same polarization behavior and the corrosion rate. The movement of the open-circuit potential first towards more negative values and later to more positive values is a phenomenon common to all kinds of specimens. This phenomenon resulted from an initial stimulation of the anodes by sulphide ions produced by the bacteria from the reduction of sulphate ions in the medium, followed by the formation of an insoluble partly protective film of ferrous sulphide on the surface of the electrode. The effect of SRB (Sulphate Reducing Bacteria) is clearly showed in the cathodic polarization curve. When the SRB is in a condition of rapid growth, there is a strong cathodic depolarization. However, as the sulphate is depleted and reaction products accumulate, the activity of the bacteria declines and the cathodic polarization curve returns the same form as in the inoculated culture.
\end{abstract}

KEY WORDS: microbiologically influenced corrosion; sulphate reducing bacteria; thermomechanical control process steel.

\section{Introduction}

Corrosion of cargo oil tank steel structures has been focused because of its severe accelerated corrosion. Recently, the OCIMF (Oil Companies International Marine Forum) member have reported that corrosion rate of double hull tanker structures less than 3 years old was as high as $0.16 \mathrm{~mm} / \mathrm{yr}$ to $0.24 \mathrm{~mm} / \mathrm{yr}$, which is approximately 2 to 3 times that which would normally be anticipated. In addition this accelerated general corrosion, an increase in the incidence and severity of pitting corrosion in lower deck steel plates of cargo tanks has been observed. Average pit depth of between 2.0 and $3.0 \mathrm{~mm}$ with a maximum pit depth of $4.0 \mathrm{~mm}$ has been reported on ships less than 2 years old. ${ }^{1)}$

This excessive pitting corrosion in the lower deck steel plates has been considered as microbial attack from bacteria, because the number of SRB was detected in the residual water of double hull tank. The higher temperature coupled with residual water of double hull tanker due to the insulating effect offers favorable condition for SRB to proliferate. When the bacteria attach on a steel surface, they can proliferate and a corrosion pit develop at the site. Pitting corrosion is characteristic of the action of the SRB on steels, with the pits being opened and filled with soft black corro- sion products in the form of the iron sulphide.

Recently, TMCP (Thermo-mechanical Control Process) steel has been widely used as ship structural steel because of its high tensile strength and excellent weldability. However, there have been rumors among ship-owners that TMCP steels are more susceptible to corrosion than other steel. In this study, the corrosion behaviors of TMCP steel have been studied to determine whether or not they differ from steels manufactured by other methods. In order to elucidate the corrosion characteristics of TMCP steels by comparison with conventional mild steel and normalized steel, immersion and electrochemical tests have been performed. Particularly, corrosion resistance of TMCP steels in an environment that includes SRB influenced corrosion has been focused.

\section{Experimental Procedures}

\subsection{Materials}

In order to examine the effect of manufacturing processes on the MIC, three kinds of specimen such as conventional rolled steel (Specimen A), TMCP steel (Specimen B) and normalized steel (Specimen C) were prepared. The chemical compositions of each specimen were listed in Table 1. 


\subsection{Bacteria and Medium}

The medium for bacteria growth was Postgate medium and had the following composition:

Basal Medium: $\mathrm{K}_{2} \mathrm{HPO}_{4} 0.5 \mathrm{~g}, \mathrm{NH}_{4} \mathrm{Cl} 1.0 \mathrm{~g}, \mathrm{CaCl}_{2} \cdot 2 \mathrm{H}_{2} \mathrm{O}$ $0.1 \mathrm{~g}, \mathrm{MgSO}_{4} \cdot 7 \mathrm{H}_{2} \mathrm{O} 2.0 \mathrm{~g}$, sodium lactate (70\%) $5.0 \mathrm{ml}$, Yeast extract $1.0 \mathrm{~g}$, Distilled water $1.0 \mathrm{~L}, \mathrm{pH} 7.4$

Solution 1: $\mathrm{FeSO}_{4} \cdot 7 \mathrm{H}_{2} \mathrm{O} 5.0 \mathrm{~g}$, Distilled water $100 \mathrm{ml}$

Solution 2: Ascorbic acid $1.0 \mathrm{~g}$, Sodium thioglycollate $1.0 \mathrm{~g}$, Distilled water $100 \mathrm{ml}$

Solution 1 of $50 \mathrm{mg}$ and solution 2 of $10 \mathrm{mg}$ were added in the basal medium of $100 \mathrm{mg}$ at a sterile condition.

The sulphate reducing bacteria was used, namely Desulfovibrio dusulfuricans (KCTC 2360), which were maintained in stock culture in the medium under an atmosphere of nitrogen. Regular checks were made on the purity of the stock cultures by the method described by Postgate. All inoculations from stock were made using an inoculum of $1 \mathrm{ml}$ for $30 \mathrm{ml}$ of sterile medium.

\subsection{Electrochemical Cell}

The working electrodes were coupons embedded in an epoxy resin. The coupons were polished to a 600 grit silicon carbide, degreased in a acetone followed by an ethanol rinse, and suspended vertically mounted on a glass tube. Electrical connection was made via a brass rod at the center

Table 1. Chemical compositions of each specimen (wt\%).

\begin{tabular}{c|ccccccccc}
\hline & $\mathrm{C}$ & $\mathrm{Si}$ & $\mathrm{Mn}$ & $\mathrm{P}$ & $\mathrm{S}$ & $\mathrm{Cu}$ & $\mathrm{Ni}$ & $\mathrm{Ti}$ & $\mathrm{V}$ \\
\hline $\mathrm{A}$ & 0.17 & 0.25 & 0.70 & 0.018 & 0.007 & 0.04 & 0.01 & 0.002 & 0.001 \\
\hline $\mathrm{B}$ & 0.11 & 0.253 & 1.29 & 0.02 & 0.005 & 0.01 & 0.01 & 0.011 & 0.002 \\
\hline $\mathrm{C}$ & 0.174 & 0.43 & 1.45 & 0.02 & 0.003 & 0.17 & 0.11 & 0.004 & 0.065 \\
\hline
\end{tabular}

and the joints sealed with a silicon rubber. The polarization cell was constructed of Pyrex glass. The working electrode and the auxiliary electrode of carbon rod were supported in a glass holder through a rubber bung in the top of the cell. The reference electrode was brought close to the surface of the working electrode by means of luggin capillary with saturated $\mathrm{KCl}$. Current was supplied to the cell by means of a EG\&G model 273 potentialstat unit and the potentials were measured against to a saturated calomel electrode (SCE). The test temperature was $40^{\circ} \mathrm{C}$.

\section{Results and Discussions}

\subsection{Microstructures}

The microstructure of steel is affected by manufacturing processes of steel plate. Figure 1 shows the microstructure of each specimen. The grain sizes of conventional rolled steel (specimen A) is much larger than those of TMCP steel (specimen B) and normalized steel (specimen C). The average grain sizes of specimen A, B and C are 16.06, 9.06 and $10.09 \mu \mathrm{m}$. The fraction, distribution, morphology, and composition of inclusions as well as grain size are important in determining the MIC response of materials. As shown in Fig. 2, all the specimens show no difference in inclusion distribution. Average inclusion size of each specimen is $1.489 \mu \mathrm{m}$ of specimen A, $1.379 \mu \mathrm{m}$ of specimen B and $1.515 \mu \mathrm{m}$ of specimen $\mathrm{C}$. The size of almost all inclusions is below $3 \mu \mathrm{m}$. Although the number of inclusions of Specimen B is larger than that of the other specimens, the area fraction of inclusions of all specimens do not show any difference with each other. From these results, it is known that TMCP steel has a finer and much inclusions than conventional rolled steel and normalized steel.

To observe the composition of inclusion, EDS analysis

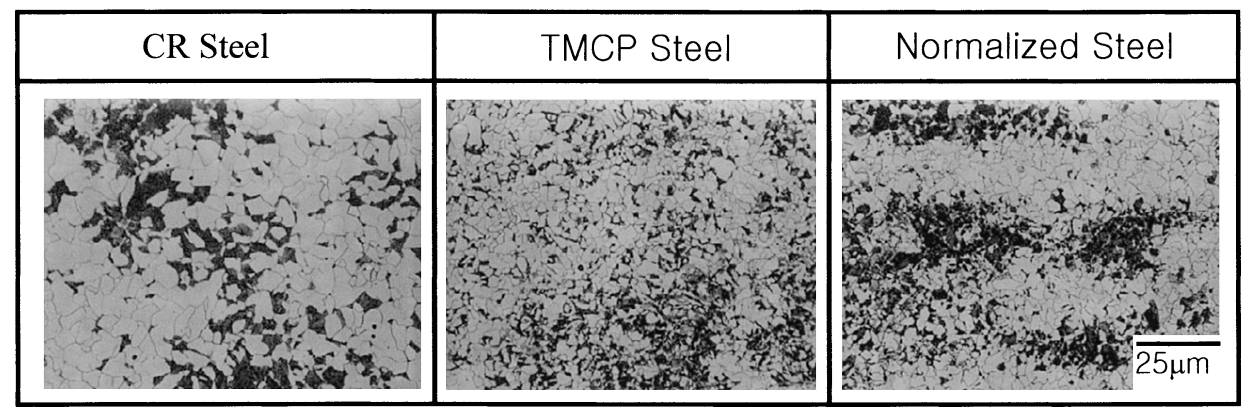

Fig. 1. Microstructure of each specimen.
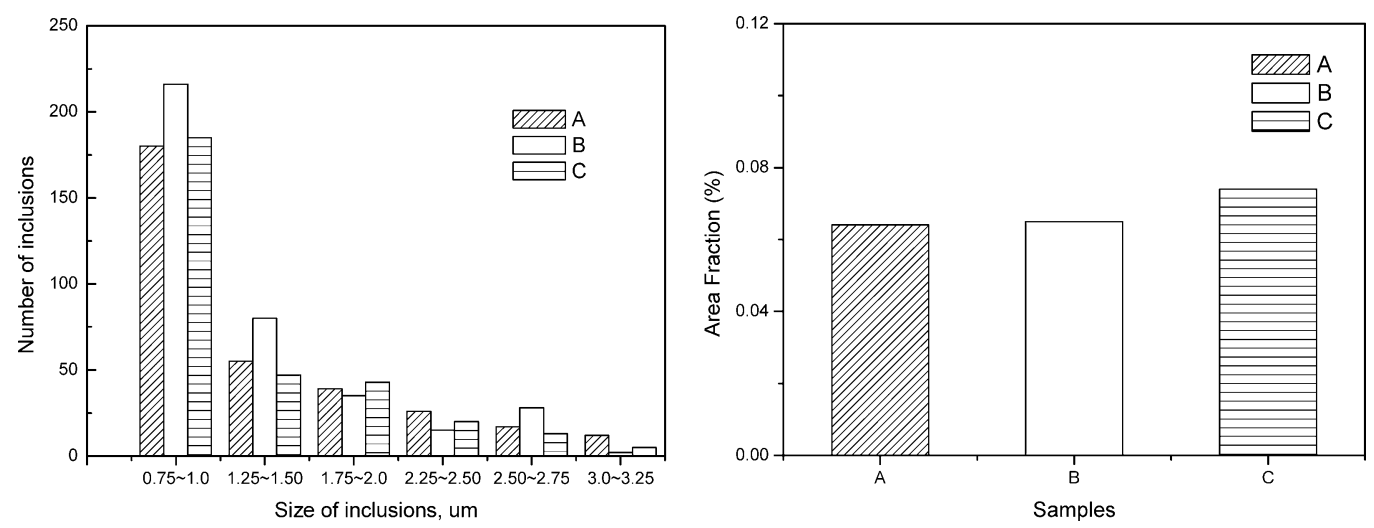

Fig. 2. Analysis of inclusions of each specimen. 


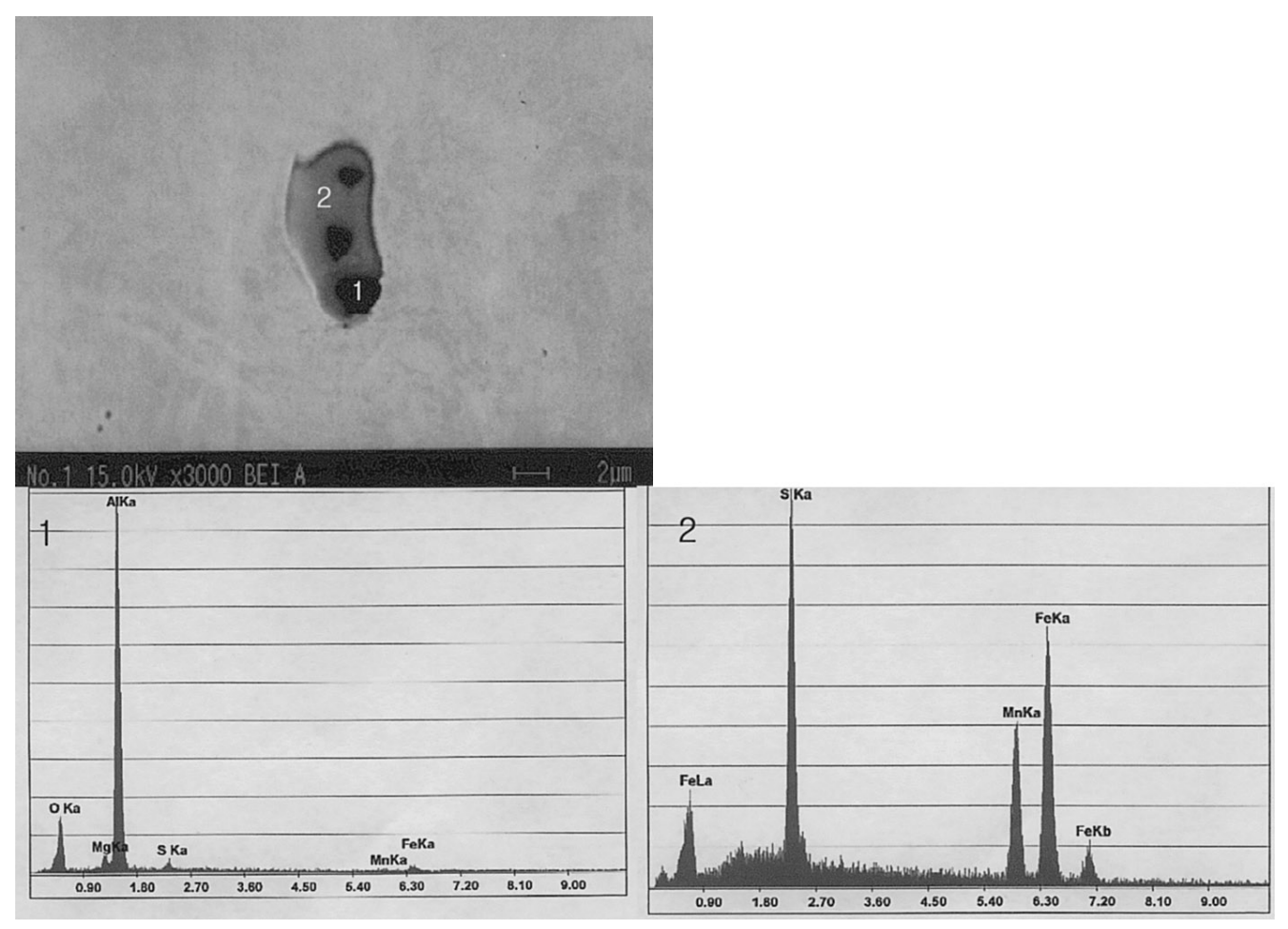

Fig. 3. SEM image and EDS analysis of inclusion.

Table 2. The number of SRB in the crude oil and sea water

\begin{tabular}{c|cccc}
\hline Sample & MIPO & SK-S & SK-B & SW \\
\hline SRB(MPN/g or ml) & 13.8 & 9.1 & 7450 & 0.15 \\
\hline \multicolumn{7}{l}{ MIPO : Tar of cargo oil tank, SK-S : Crude oil of cargo oil tank, } \\
SK-B : Water at the bottom of cargo oil tank, SW : Sea water
\end{tabular}

was performed. Inclusions of all specimens show spherical and cylindrical shape and consist of gray part and black part as shown in Fig. 3. The gray part is aluminum oxide, and the black part surrounded by gray part is manganese sulphide. Regardless of size, most of inclusions are the mixture of $\mathrm{MnS}$ and $\mathrm{Al}_{2} \mathrm{O}_{3}$.

\subsection{Existence of Bacteria in the Cargo Oil Tank}

In order to identify the existence of bacteria causing MIC of cargo oil tank, crude oil, tar of cargo oil tank, water at the bottom of cargo oil tank, and sea water were analyzed. In crude oil, any bacteria are not detected. However, sludge and fluid remaining at the bottom of the cargo oil tank contains SRB. Table 2 lists the number of SRB detected in the sludge and fluid remaining at the bottom of the cargo oil tank. The number of SRB detected in the tar of crude oil is $13.8 \mathrm{MPN} / \mathrm{g}$. The fluids gathering at the bottom of cargo oil tank are separated two layer after $24 \mathrm{~h}$. The upper layer seems to be a crude oil and the lower layer seems to be a sea water. The number of SRB detected in the upper layer and the lower layer is 9.1 and $7450 \mathrm{MPN} / \mathrm{ml}$. From a detection of large number of SRB in the lower layer of fluid, SRB detected in the cargo oil tank is considered to come from the seawater. To confirm this, seawater gathered at sea near the Pusan was analyzed. Although anaerobic bacteria SRB do not grow in the seawater that contains oxygen, the small number of SRB is detected in the seawater. It is reported that a small number of SRB living in the sediments of sea can exist in the seawater because of the difference
Table 3. The number of SRB at each culturing condition (MPN/ml).

\begin{tabular}{l|llll}
\hline & 1 Month & 2 Month & 3 Month & 4 Month \\
\hline $\mathrm{A}$ & $9.3 \times 10^{5}$ & $1.5 \times 10^{6}$ & $6.4 \times 10^{7}$ & $4.4 \times 10^{7}$ \\
\hline $\mathrm{B}$ & $4.6 \times 10^{6}$ & $7.3 \times 10^{6}$ & $4.6 \times 10^{8}$ & $3.5 \times 10^{7}$ \\
\hline $\mathrm{C}$ & $9.3 \times 10^{5}$ & $1.1 \times 10^{6}$ & $4.6 \times 10^{8}$ & $2.1 \times 10^{8}$ \\
\hline
\end{tabular}

between the rise and fall of the tide. ${ }^{2)}$ Because the seawater is used to clean the cargo oil tank, a small number of SRB contained in the seawater can cultivate to large number of SRB as shown in Table 2.

In order to investigate the cultivation rate of SRB, the number of SRB measured in the postgate medium containing specimen with time. Table 3 lists variations of the number of SRB with time. An initial number of SRB inoculated at each specimen was $6.8 \times 10^{4}$. The number of SRB was increased for 3 months, however, the number of SRB was not increased any more after 3 months. From these results, it is considered that a small number of SRB can cause the MIC through the cultivation for long time if the environment can satisfy the condition to live SRB.

\subsection{Immersion Test}

To evaluate the resistance to MIC of each specimen, immersion test was performed. Specimens were immersed in the culture medium containing SRB, and weight changes of specimens were measured for 5 months. The temperature was maintained at $40^{\circ} \mathrm{C}$ during the immersion test. Specimens extracted after 1 month were covered over the entire surface with a soft black coating which was weakly adherent. On the other hand, specimens extracted after 5 months were covered with a similar soft coating, but underlying this were irregular patches of a gray compact and very adherent layer. The specimens were washed after examination and the corrosion product removed by immersion in 
acid solution. The corrosion rate in $\mathrm{mm} / \mathrm{yr}$ may be calculated from

$$
\text { Corrosion rate }(\mathrm{mm} / \mathrm{yr})=(87.6 \mathrm{~W}) /(D A T)
$$

Where $W$ is weight loss in milligrams, $D$ is density in grams per cubic centimeter, $A$ is area in square centimeters, and $T$ is the time in hours. Figure 4 shows the corrosion rate of each specimen in the culturing medium containing SRB. Regardless of kind of specimen, the corrosion rate is decreased with increasing the time. Although the number of $\mathrm{SRB}$ is increased as listed in Table 3, the corrosion rate is decreased with increasing the time. This decrease in corrosion rate is related with formation of corrosion products formed on the surface of specimen which are prohibiting the transport of ionic species. For 1 month, the corrosion rate of TMCP steel is higher than that of other specimen. However, the corrosion rates of all specimens do not show any difference after 2 months. From these results, it is considered that the resistance to MIC is not affected by the number of SRB and by the manufacturing processes of steel.

\subsection{Corrosion Potential}

Measurement of the corrosion potential is the easiest electrochemical method which is providing the least amount of mechanistic information. Figure 5 shows the variation of corrosion potential of each specimen with time in sterile culturing medium and culturing medium containing SRB. In sterile medium, the corrosion potential of each specimen is decreased with increasing the time. However, the range of potential variation is below $10 \mathrm{mV}$ for $140 \mathrm{~d}$. This small variation is considered to belong to the experimental error. The corrosion potential of each specimen is maintained for long time in sterile culturing medium. On the other hand, the corrosion potential of each specimen moves first towards more negative values and later to more positive values in culturing medium containing SRB. This result is in good agreement with other researcher's result. ${ }^{3)}$ This phenomena was interpreted as being due to an initial stimulation of the anodes by sulphide ions produced by the bacteria from the reduction of sulphate ions in the medium, followed by the formation of an insoluble partly protective film of ferrous sulphide on the surface of the electrode.

\subsection{Polarization Curve}

From the corrosion rate calculated from weight loss and the corrosion potential, it is observed that corrosion resistance of conventional rolled steel, TMCP steel, and normalized steel under SRB environments are very similar. Therefore, in order to investigate the effect of SRB on the corrosion behavior of $\mathrm{C}-\mathrm{Mn}$ steels, the potentiodynamic technique is used. Figure 6 shows the polarization curves of conventional rolled steel in different electrolytes with and without SRB. In a culturing medium without SRB, a slight fall in corrosion potential with time was observed amounting to $30 \mathrm{mV}$. This slight fall of corrosion potential has been reported by other researchers and may be ascribed to the scavenging by the steel of the last traces of oxygen from the electrolytes. On the other hand, the corrosion potential was increased considerably after $3 \mathrm{~d}$. However, the corrosion potential was not increased any more after $7 \mathrm{~d}$.

In the potentiodynamic techniques, the Tafel technique is

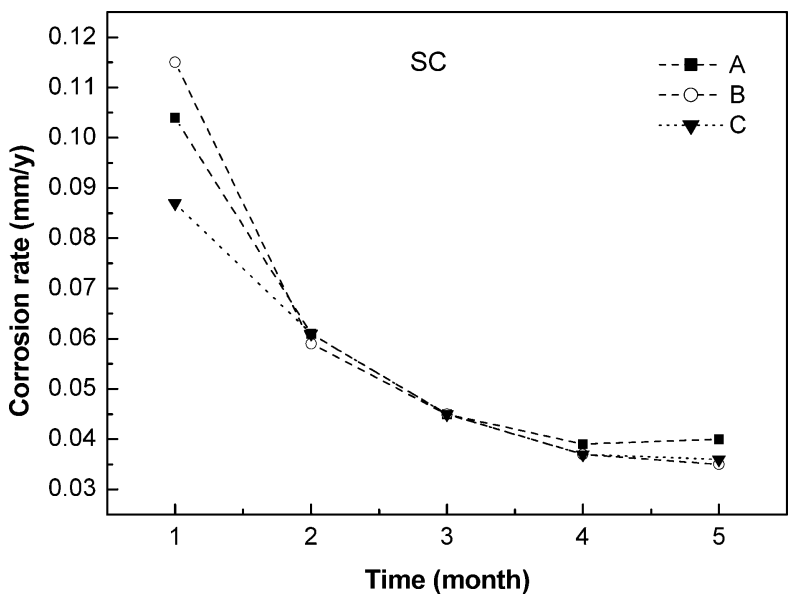

Fig. 4. Corrosion rate of each specimen in the SRB culturing medium.
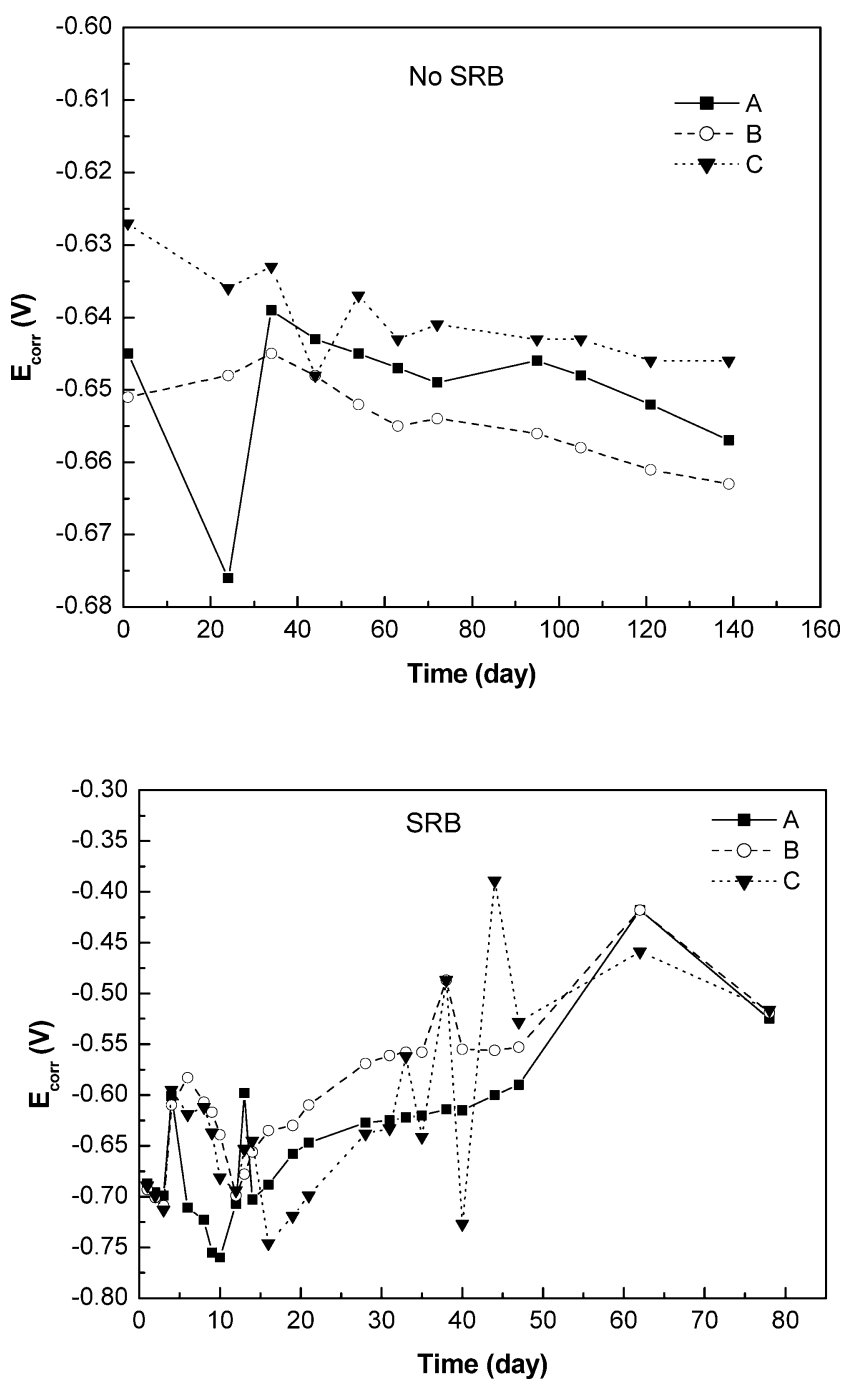

Fig. 5. Corrosion potential of each specimen in culturing medium with and without SRB.

used to obtain an accurate estimate of the corrosion rate of a metal in an electrolyte. A Tafel plot is performed to experimentally determine corrosion current $\left(i_{\text {corr }}\right)$ from which the corrosion rate is calculated. To compare the corrosion rate of conventional rolled steel and TMCP steel, corrosion currents were determined from the polarization curves 

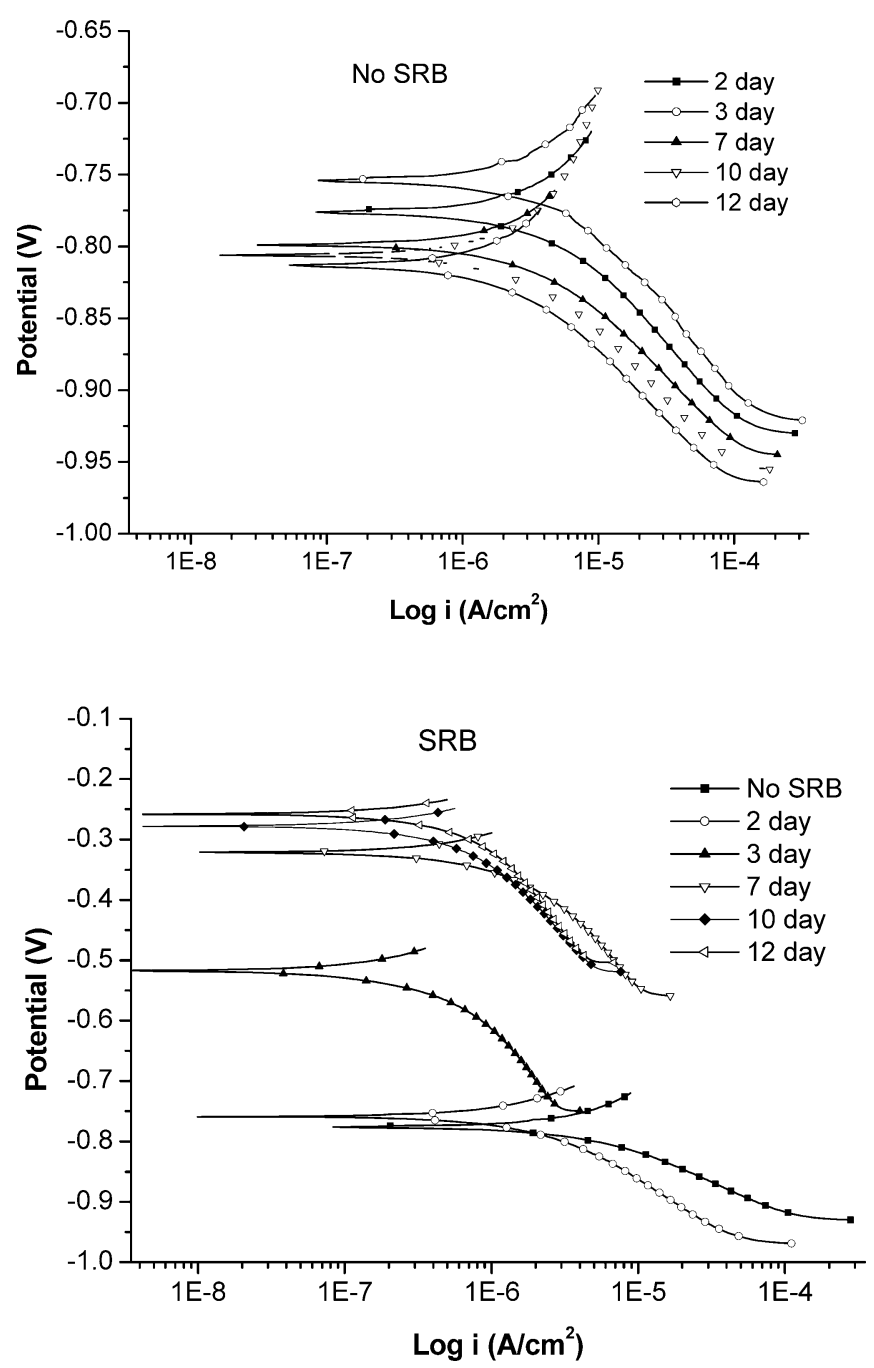

Fig. 6. Polarization curve of $\mathrm{CR}$ steel in culturing medium with and without SRB.

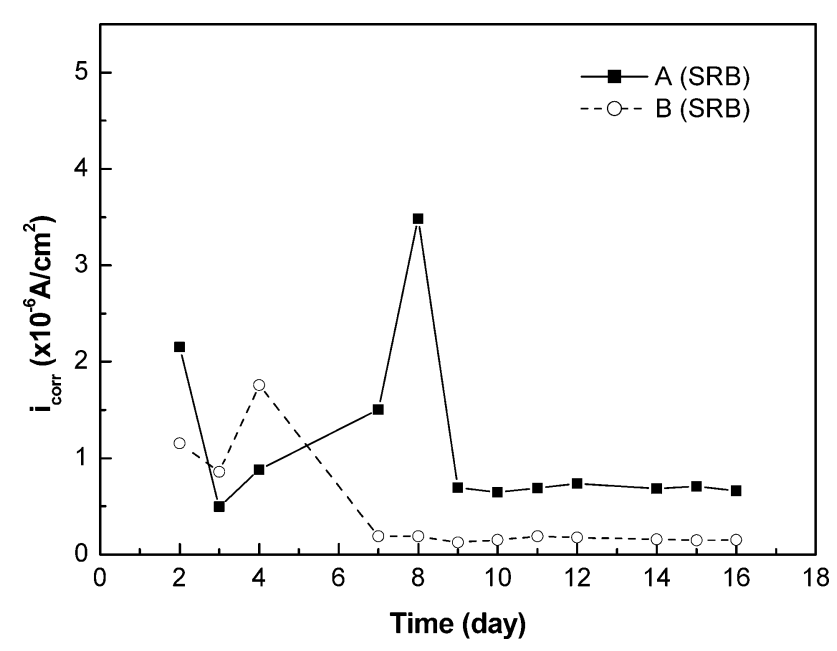

Fig. 7. Corrosion current of each specimen in culturing medium with and without SRB.

through Tafel plots. Figure 7 shows the corrosion currents of each specimen with time in a culturing medium with $\mathrm{SRB}$. The corrosion current of conventional rolled steel is lower than that of TMCP steel. Namely, TMCP steel is not more sensitive to MIC than conventional rolled steel.

Several mechanisms suggested by so many researchers to

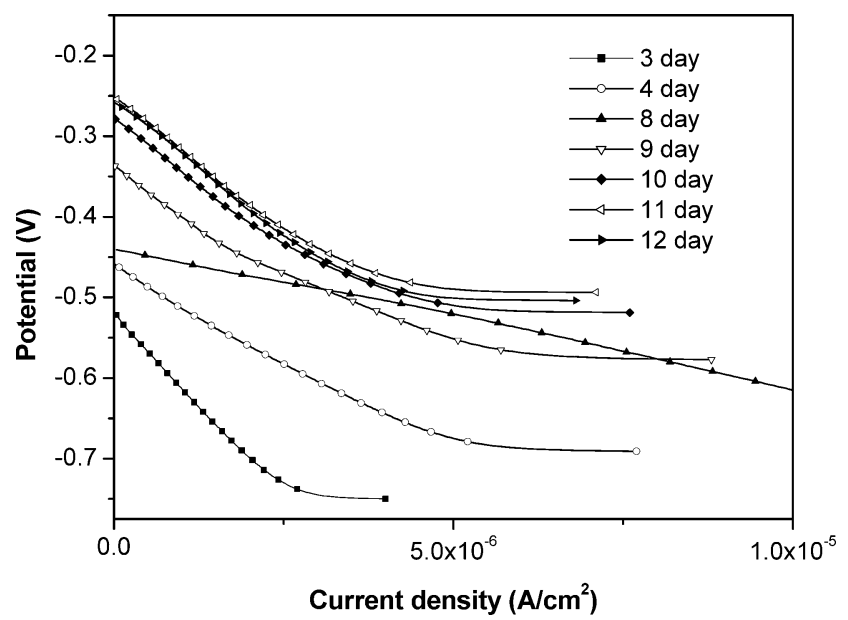

Fig. 8. Cathodic polarization curve of conventional rolled steel in culturing medium with SRB.

explain corrosion behavior in terms of involvement of the SRB. $^{3-9)}$ It was clear that SRB performed a type of anaerobic respiration in reducing sulphate to sulphide. Kuhr and Vlugt suggested that the SRB utilize the cathodic hydrogen for the dissimilatory reduction of sulphate according to the equations given below.

$$
\begin{aligned}
& 4 \mathrm{Fe} \rightarrow 4 \mathrm{Fe}^{2+}+8 \mathrm{e}^{-} \quad \text { anodic reaction } \\
& 8 \mathrm{H}_{2} \mathrm{O} \rightarrow 8 \mathrm{H}^{+}+8 \mathrm{OH}^{-} \quad \text { dissolution of water } \\
& 8 \mathrm{H}^{+}+8 \mathrm{e}^{-} \rightarrow 8 \mathrm{H} \quad \text { cathodic reaction } \\
& \mathrm{SO}_{4}^{2-}+8 \mathrm{H}^{+} \rightarrow \mathrm{S}^{2-}+4 \mathrm{H}_{2} \mathrm{O} \\
& \text { cathodic depolarization by bacteria } \\
& \mathrm{Fe}^{2+}+\mathrm{S}^{2-} \rightarrow \mathrm{FeS} \quad \text { corrosion product } \\
& 3 \mathrm{Fe}^{2+}+6 \mathrm{OH}^{-} \rightarrow 3 \mathrm{Fe}(\mathrm{OH})_{2} \quad \text { corrosion product } \\
& 4 \mathrm{Fe}+\mathrm{SO}_{4}^{2-}+4 \mathrm{H}_{2} \mathrm{O} \rightarrow 3 \mathrm{Fe}(\mathrm{OH})_{2}+\mathrm{FeS}+2 \mathrm{OH}^{-} \\
& \text {overall reaction }
\end{aligned}
$$

In order to observe the cathodic depolarization by SRB, cathodic polarization curves are plotted as shown in Fig. 8. The effect of SRB able to utilize hydrogen is clearly shown in Fig. 8. After $4 \mathrm{~d}$ incubation, the slope of curve are started to change, and after $8 \mathrm{~d}$ incubation, there is a strong cathodic depolarization. As the sulphate is depleted in the culturing medium and reaction products accumulate, the activity of SRB declines and the cathodic polarization curve returns after $10 \mathrm{~d}$ to much the same form as in the $4 \mathrm{~d}$ inoculation. The cathodic depolarization is a function of the activity. Booth and Tiller are reported that the cathodic depolarization is restored with the fresh medium. ${ }^{3)}$

\subsection{Corrosion Product}

Surface and cross-sectional image of the corrosion product formed on the each specimen was observed after immersion test. Figure 9 shows surface and cross-sectional images in the culturing medium without SRB after 3 months. The corrosion products formed on the all specimens have the flat surfaces and the same images. This corrosion product is considered to be $\mathrm{Fe}_{2} \mathrm{P}$ through the EDS analysis as shown in Fig. 10. On the other hand, the protrusions are observed on the surface, and the interfaces of corrosion product and base metal are not flat after immersion test for 3 months in culturing medium with SRB as shown 


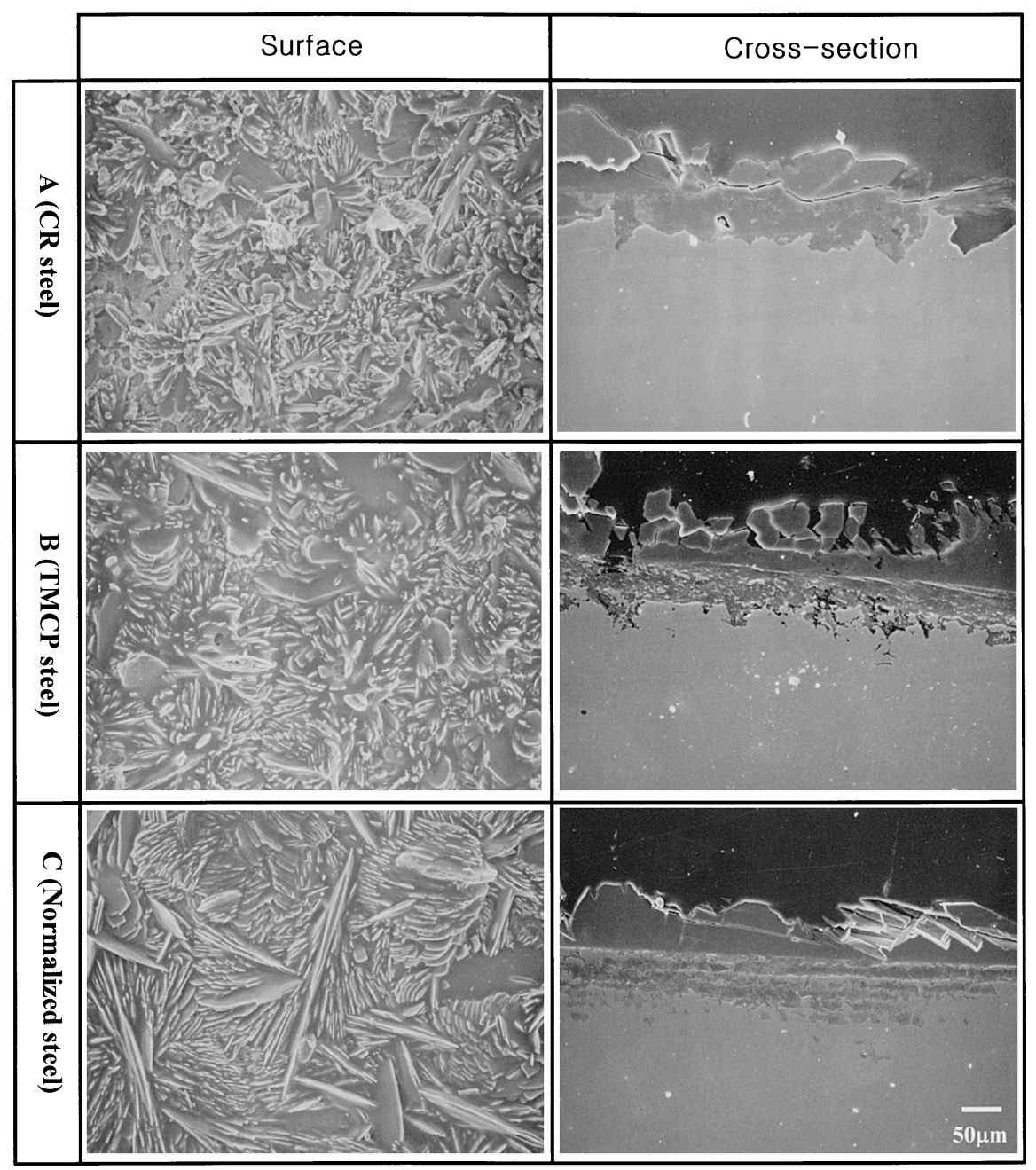

Fig. 9. Surface and cross-sectional images of each specimen after immersion test for 3 months in the culturing medium without SRB.

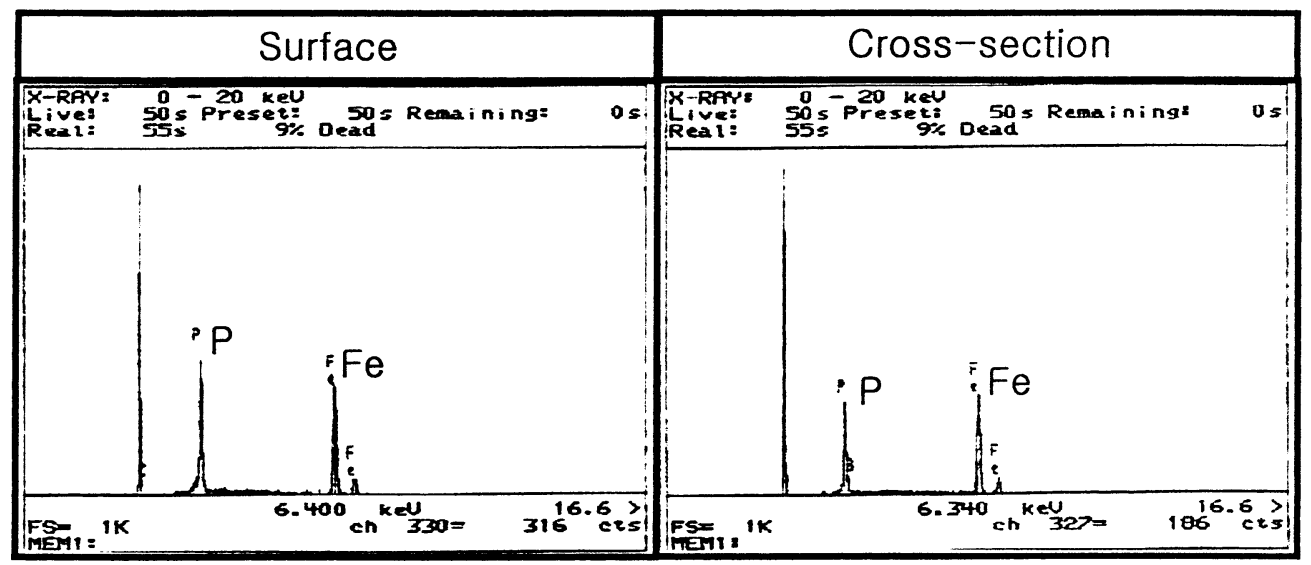

Fig. 10. EDS analysis of corrosion product formed on the B (TMCP steel) specimen after immersion test for 3 months in the culturing medium without SRB.

in Fig. 11. The sites of protrusions are good agreement with those of deeper corrosion depth. This localized corrosion with associated tubercles is one of the basic forms of MIC. The depths at localized corrosion site are 30-35 $\mu \mathrm{m}$ of conventional rolled steel, $20-25 \mu \mathrm{m}$ of TMCP steel, and $30-35 \mu \mathrm{m}$ of normalized steel. As explained in previous chapter corrosion product is the result of the interaction between anodic reaction and environment $\left(\mathrm{Fe}^{2+}+\mathrm{S}^{2-} \rightarrow \mathrm{FeS}\right)$ or between anodic and cathodic reaction $\left(3 \mathrm{Fe}^{2+}+6 \mathrm{OH}^{-} \rightarrow\right.$ $\left.3 \mathrm{Fe}(\mathrm{OH})_{2}\right)$. To identify the corrosion product, EDS analysis was performed as shown in Fig. 12. In the surface of corrosion product, $\mathrm{S}$ is detected, but $\mathrm{S}$ is not detected in the inner part of corrosion product. From these results, it is considered that corrosion products consists of $\mathrm{FeS}$ of the outer side and $\mathrm{Fe}(\mathrm{OH})_{2}$ of the inner side. 


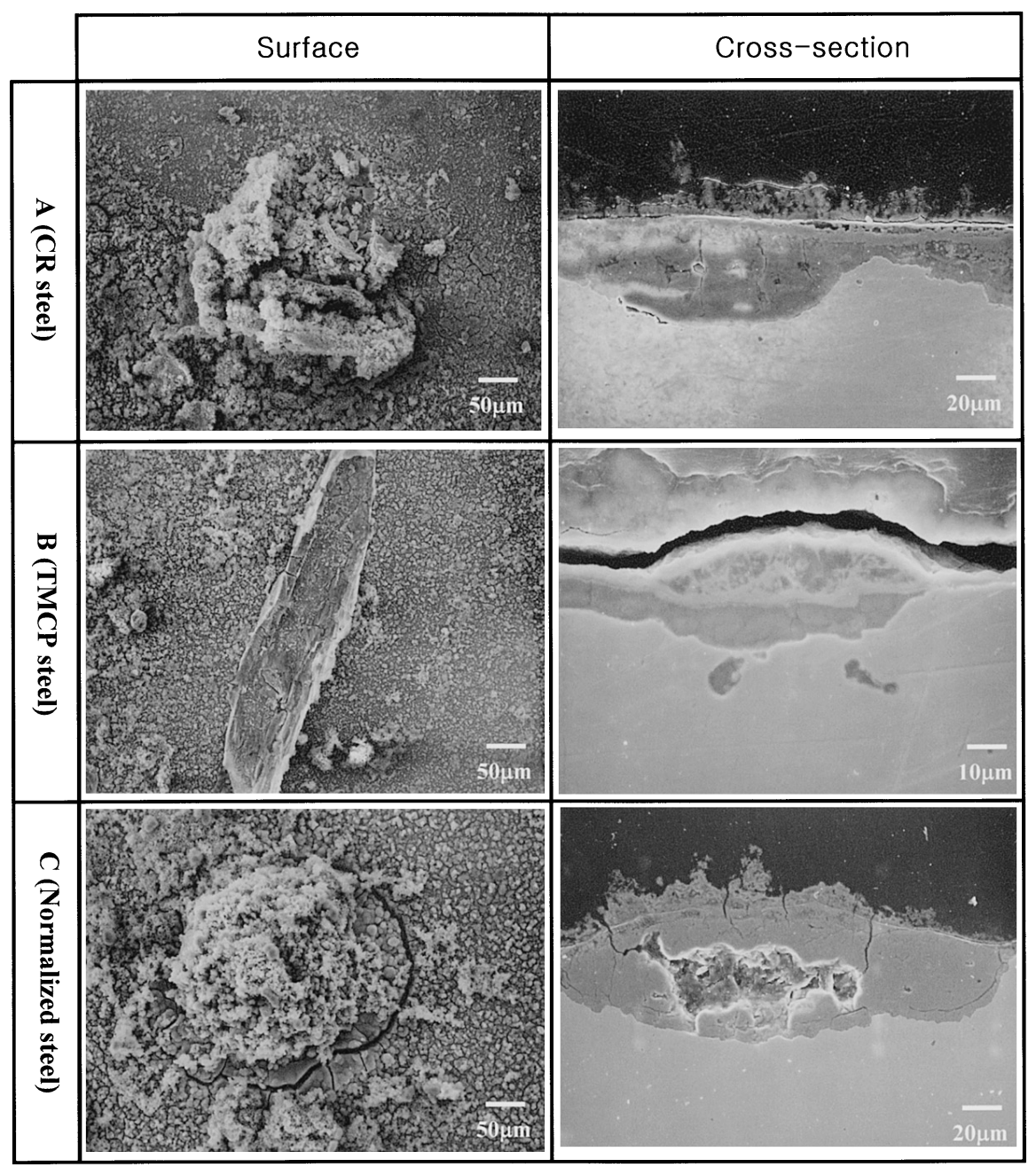

Fig. 11. Surface and cross-sectional images of each specimen after immersion test for 3 months in the culturing medium with SRB.

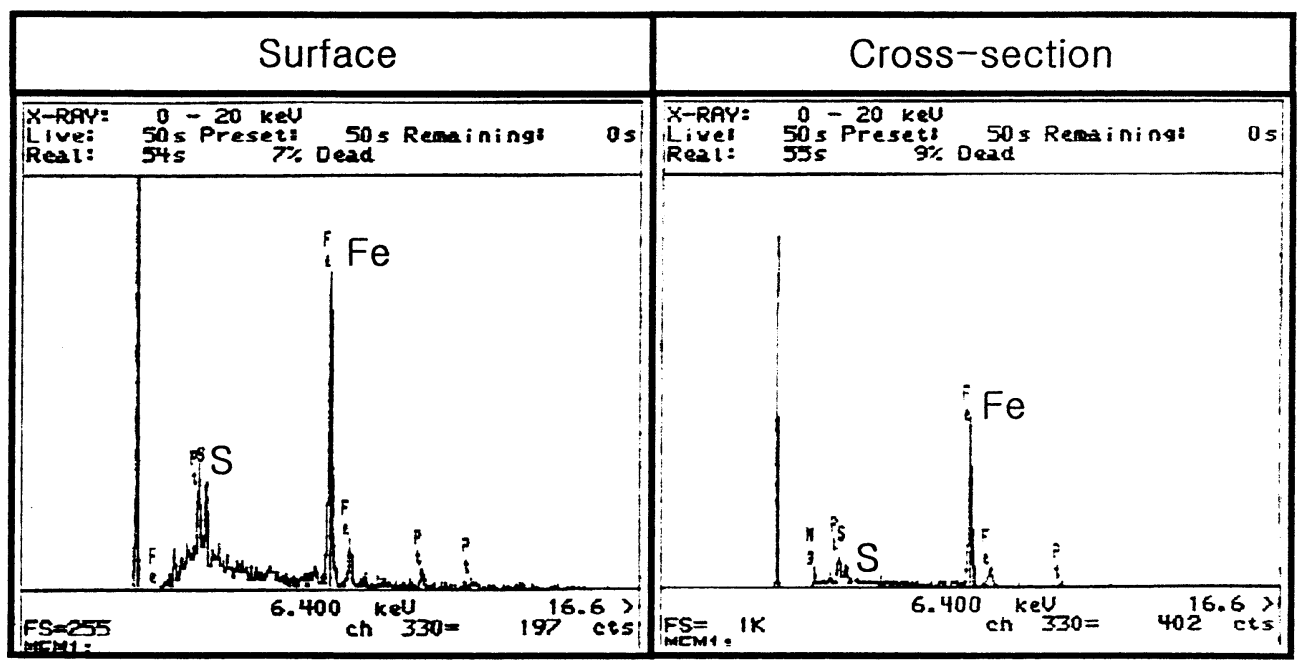

Fig. 12. EDS analysis of corrosion product formed on the B (TMCP steel) specimen after immersion test for 3 months in the culturing medium without SRB.

\section{Conclusions}

(1) Although anaerobic bacteria SRB do not grow in the seawater that contains oxygen, the small number of $\mathrm{SRB}$ is detected in the seawater. Because a small number of SRB can grow the large number of SRB through the culti- vation for long time if the environment can satisfy the condition to live SRB, a small number of SRB contained in the seawater can cause the MIC of cargo oil tank.

(2) From the corrosion rates calculated from the immersion tests and corrosion current densities determined from the polarization curves, it is confirmed that TMCP 
steel is not more sensitive to MIC than conventional rolled steel and normalized steel. The corrosion rates calculated after immersion tests for 5 months are $0.035 \mathrm{~mm} / \mathrm{yr}$ of conventional rolled steel, $0.032 \mathrm{~mm} / \mathrm{yr}$ of TMCP steel and $0.031 \mathrm{~mm} / \mathrm{yr}$ of normalized steel.

(3) The corrosion potential of each specimen is maintained for long time in a culturing medium without SRB. On the other hand, the corrosion potential of each specimen moves first towards more negative values and later to more positive values in culturing medium with SRB. This phenomena was interpreted as being due to an initial stimulation of the anodes by sulphide ions produced by the bacteria from the reduction of sulphate ions in the medium, followed by the formation of an insoluble partly protective film of ferrous sulphide on the surface of the electrode.

(4) The mechanism of MIC is cathodic depolarization. The essential role of SRB is the removal of hydrogen in re- ducing sulphate to sulphide. However, as the sulphate is depleted in the culturing medium and reaction products accumulate, the activity of SRB declines and the cathodic polarization does not proceed any more.

\section{REFERENCES}

1) OCIMF Report, September (1997).

2) G. Rheinheimer: Aquatic Microbiology, 2nd ed., John Wiley \& Sons, (1992).

3) G. H. Booth and A. K. Tiller: Trans. Faraday Soc., (1960), 1989.

4) M. Stephenson and M. Stickland: Biochem. J., 25 (1931), 215.

5) C. A. H. von Wolzogen Kuhr and L. S. van der Vlurt.: Water, 18 (1934), 147.

6) F. Wormwell and T. W. Farrer: Chem. Ind., (1952), 108.

7) J. N. Wanklyn and C. J. P. Spruit: Nature (Lond.), 169 (1952), 928.

8) M. Solti and J. Horvath: Werkst. Korros., 9 (1958), 283.

9) T. P. Hoar and T. W. Farrer: Corros. Sci., 1 (1961), 49. 